\title{
Fetoscopic Amniotic Band Release in a Case of Chorioamniotic Separation: An Innovative New Technique
}

Michael A. Belfort, MBBCH, MD, PhD ${ }^{1}$ William E. Whitehead, MD ${ }^{2}$ Robert Ball, MD ${ }^{3}$ Robert Silver, MD Alireza Shamshirsaz, MD ${ }^{2}$ Rodrigo Ruano, MD, PhD ${ }^{2}$ Jimmy Espinoza, MD ${ }^{2}$ Judith Becker, MD $^{5}$ Olutoyin Olutoye, $\mathrm{MD}^{6}$ Larry Hollier, $\mathrm{MD}^{7}$

${ }^{1}$ Department of Obstetrics and Gynecology, Texas Children's Hospital Fetal Center, Houston, Texas

2 Department of Neurosurgery, Texas Children's Hospital Fetal Center, Houston, Texas

${ }^{3}$ St. Mark's Hospital, Salt Lake City, Utah

${ }^{4}$ Department of Obstetrics and Gynecology, University of Utah, Utah

${ }^{5}$ Department Pediatrics (Cardiology), Texas Children's Hospital Fetal Center, Houston, Texas

${ }^{6}$ Department Anesthesiology, Texas Children's Hospital Fetal Center, Houston, Texas

${ }^{7}$ Department Surgery (Plastic Surgery), Texas Children's Hospital Fetal Center, Houston, Texas

Am J Perinatol Rep 2016;6:e222-e225.
Address for correspondence Michael Belfort, $\mathrm{MBBCH}, \mathrm{MD}, \mathrm{PhD}$, Department of Obstetrics and Gynecology, Office of Chairman F1096, Baylor College of Medicine, 6651 Main Street, Houston, Texas, 77030 (e-mail: Belfort@bcm.edu).

\begin{abstract}
Introduction Fetoscopic release of amniotic bands has proved its life- and limb-saving potential. Rupture of the amnion and separation of chorion from the amnion and uterine wall can however preclude the standard fetoscopic approach to release the amniotic bands using a single port.

Methods and Materials A 28-year-old G1P0 woman was referred to our unit at 19 weeks due to amniotic band syndrome involving the left ankle, the infrapatellar region of the right leg, and the umbilical cord. Of note, part of the fetus was seen outside the amniotic cavity by ultrasonography and the left ankle and foot were severely swollen. The patient underwent a laparotomy and fetoscopic release of the amniotic bands as well as partial amnionectomy using two uterine ports and $\mathrm{CO}_{2}$ as distention.
Keywords
- fetus
- amniotic band
$-\mathrm{CO}_{2}$
- 2-port
- simplified closure weeks the patient went into labor, which was augmented resulting vaginal delivery of a 2,460 -g male infant. The infant was noted to have a shallow skin indentation on the left lower extremity near the ankle. The infant was discharged in excellent condition. Conclusion In those cases where release of an amniotic band is impossible due to membrane separation, surgery in a $\mathrm{CO}_{2}$-filled uterus offers an option.
\end{abstract}

\section{Case Report}

A 28-year-old gravida 1 para 0 patient was referred to the Fetal Center at Texas Children's Hospital at 19 weeks of

received

November 13, 2015

accepted after revision

December 17, 2015
DOI http://dx.doi.org/

$10.1055 / \mathrm{s}-0036-1579538$. ISSN 2157-6998. gestation due to the suspicion of preterm premature rupture of the membranes (PROM) and extensive chorioamniotic membrane separation. An amniotic band syndrome was also noted involving the left ankle, the infrapatellar region
Copyright $\odot 2016$ by Thieme Medical Publishers, Inc., 333 Seventh Avenue, New York, NY 10001, USA. Tel: +1(212) 584-4662.
License terms

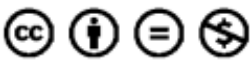




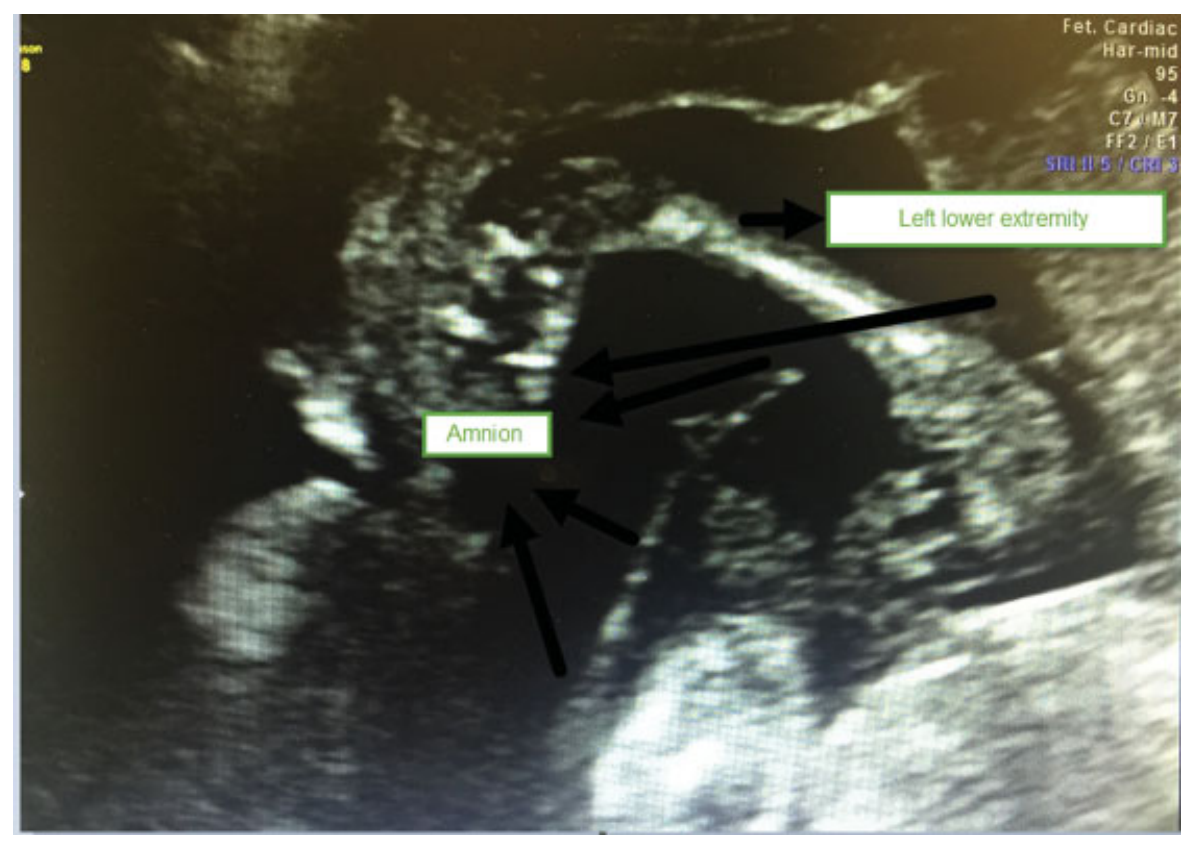

Fig. 1 The figure shows the significant swelling of the left lower extremity of the fetus prior to surgery with the lower ankle and foot clearly seen outside of the amniotic membrane.

of the right leg, and the umbilical; in addition, part of the fetus was seen outside the amniotic cavity, suggesting a significant amniotic membrane rupture ( - Fig. 1). The left ankle and left foot of the fetus were severely swollen and amniotic membrane was seen encircling the right leg attaching the umbilical cord to the fetal leg. By comparing with the result of a scan done a week earlier, there was significant worsening of the swelling of the left ankle and foot. Following ultrasonography examination and plastic surgeon consultation, the patient and her husband were offered the options of termination of pregnancy, expectant management with a significant risk for autoamputation of both fetal legs and fetal death from cord strangulation, or to follow a step-wise approach to try fetal surgery using the conventional approach and, if the visualization of the fetal structure was poor due to the nature and severity of the chorioamniotic separation, to try an experimental surgical approach to improve the visualization of the fetal structures and amniotic membranes. Following extensive consultation, the parents signed a written consent form to follow the step-wise approach which was approved by the Baylor College of Medicine Fetal Therapy Board.

At $19^{-3 / 7}$ weeks of gestation, the patient underwent laparotomy using a Pfannenstiel skin incision under general anesthesia, and following opening of the abdominal cavity the uterus was exteriorized and the placenta was mapped under ultrasound guidance. An 18G needle was then advanced through the uterine wall under sonographic guidance and then into the area between the separated chorion and amnion. A $12 \mathrm{~F}$ vascular cannula (Cook, Bloomington, IN) was then inserted into this space using Seldinger technique and sutured in place with two 4/0 Monocryl sutures. A Storz pediatric cystoscope (Storz, Tuttlingen, Germany) was then introduced into the $12 \mathrm{~F}$ cannula. The right leg was outside the amniotic cavity; in addition, the amniotic membrane was seen wrapping around the right leg and the umbilical cord. Storz hysteroscopic scissors of $1 \mathrm{~mm}$ size were then introduced into the operating channel of the cystoscope and an attempt was made to cut the membranes. This was not possible as the membranes were very mobile and we were unable to exert any countertraction to enable cutting of the membranes. The situation was further hampered by the very narrow field of view through the cystoscope because of its use in amniotic fluid. At this point, the cystoscope was withdrawn into the space between the chorion and uterine wall and approximately $60 \mathrm{~mL}$ of amniotic fluid was removed and replaced with $\mathrm{CO}_{2}$ gas $(0.5 \mathrm{~L} / \mathrm{min}$ with a maximum pressure of $12 \mathrm{~mm} \mathrm{Hg}$ ). The separated chorionic membrane was noted to be thickened and opaque. A second $12 \mathrm{~F}$ port was then inserted under direct visualization and anchored as described earlier. A 1-mm Storz hysteroscopy grasper was placed down into the operating channel of the cystoscope and used to stabilize the chorionic membranes while a 3-mm Storz laparoscopy scissors, placed through the second port, was used to make an approximately $2 \mathrm{~cm}$ opening in the chorion through which the fetus was clearly visible. Additional amniotic fluid was removed and replaced with $\mathrm{CO}_{2}$ to allow excellent visualization of the fetus. The left ankle and foot were seen to be significantly swollen below a thin amniotic band that had encircled the ankle and cut through the skin close to the bone. This band was cut with the scissors, removed from around the ankle, and then cut out and removed from the uterus. The right leg was then dissected free from a large piece of amnion that was wrapping around the leg along with a loop of umbilical cord. The piece of amnion was then removed (-Fig. 2). The fetal cardiac function evaluated by a pediatric cardiologist remained stable during the surgical procedure. Cardiac function was intermittently monitored during the procedure (at least every 10 


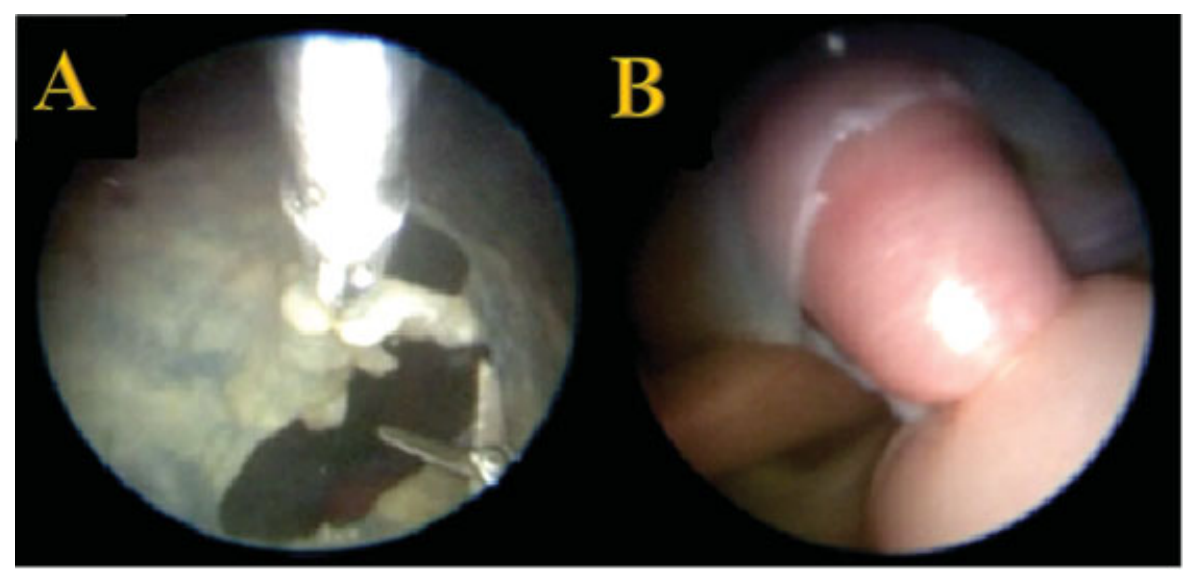

Fig. 2 Fetoscopic surgery using $\mathrm{CO}_{2}$. (A) Views of the fetoscopic procedure demonstrating the partial amnionectomy and (B) the amniotic band around the left ankle and the depth that the amniotic band had cut into the soft tissue of the ankle.

minutes) by a pediatric cardiologist using a sterile ultrasound probe placed directly onto the uterus to image the fetal heart. Normal cardiac function was assessed by heart rate, degree of triscuspid and/or mitral regurgitation, and by the ejection fraction and motion of the heart. There were no signs of maternal hypercarbia (no elevated End-tidal $\mathrm{CO}_{2}$ ) and no evidence of heart rate decelerations or bradycardia in fetus. At the completion of the surgery, the gas was slowly removed and replaced with warmed saline, the ports were removed, the uterine openings were closed with 2/0 Vicryl sutures, the uterus was returned to the abdomen, and the patient's abdomen was closed. The recovery period was as uneventful and the patient was discharged home at 72 hours postsurgery. Follow-up scans were done on a weekly basis at her local hospital. At $29^{2 / 7}$ weeks ( $\sim 10$ weeks after surgery), the patient presented with mild vaginal bleeding, oligohydramnios, and suspicion of preterm premature rupture of membranes and was admitted for inpatient management. At $34^{-1 / 7}$ weeks, the patient went into labor and delivered vaginally a 2,460-g male infant. Apgar scores were 7 at both 1 and 5 minutes of life. The neonatal course was relatively unremarkable. There was mild respiratory disease of prematurity which required mechanical ventilation for an hour as well as some oxygen therapy. However, there was no evidence of pulmonary hypoplasia. The infant also was treated for possible infection (however, the blood/urine cultures were negative) and hyperbilirubinemia. An amputation of the distal portion of the right ring finger was seen at the time of delivery as well as a shallow skin indentation on the left lower extremity near the ankle without involvement of the muscle or bone (-Fig. 3). Blood flow to the foot was excellent. The infant also had club feet. The plan is to consider minor plastic surgery for the constriction at 6 months of life. The infant was discharged in good condition on room air at 30 days of life.

\section{Discussion}

We have previously reported on the use of fetoscopic resection in amniotic fluid in cases complicated by amniotic bands, which are known to threaten the integrity of limbs as well as to increase the risk for fetal death. ${ }^{1}$ In the latter report, a review of the literature was done providing evidence that fetoscopic release of amniotic bands results in the preservation of limb function in $50 \%$ of cases. The current case is rather unique in that there was complete chorion amnion separation and the fetus was partially located outside the amniotic which precluded the use of a single or double port as has previously been reported ("under water" approach). ${ }^{2}$ The introduction of $\mathrm{CO}_{2}$ into the uterus allowed clear visualization of the chorion and the defect in the amniotic membrane through which the fetoscope had been passed. The defect was widened using laparoscopic scissors to allow for an excellent visualization of the fetus under its amniotic "shroud." In addition, this approach enabled easy manipulation of the fetus and allowed for the thick amniotic band to be removed from around the left ankle and the from below the right knee with precision. The wide field of view and the clear visualization were crucial in the success of this experimental approach, which could not have been completed using the conventional approach.

There are several insights gained from this case. First, despite a significant lysis of amniotic bands and partial amnionectomy, the patient continued pregnant without

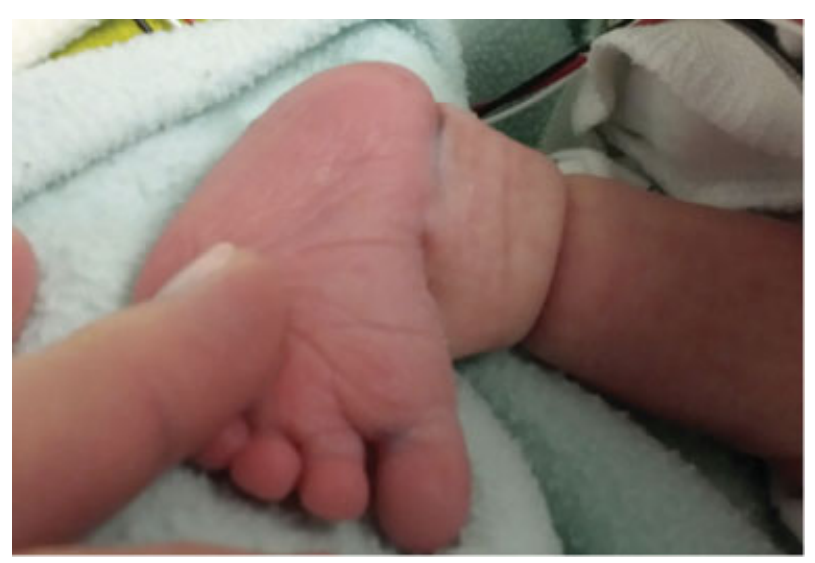

Fig. 3 Left ankle at birth. 
gross premature rupture of membranes. It is possible that amniotic fluid reaccumulation was enough to enable lung development despite preterm PROM at 19 weeks of gestation. We propose that in cases of amniotic band syndrome where single port in-fluid fetoscopy is not possible, fetoscopic surgery using $\mathrm{CO}_{2}$ as a distention media in the uterus could significantly improve the visualization and increase the chances for surgical release of the amniotic bands.

Second, despite obvious resolution of the edema in the left lower extremity, the fetus had a circumferential scar noted at the time of delivery. Although this scar appears to be mainly cosmetic, and blood flow and function in the ankle and foot were normal, the parents should be aware that surgical release of amniotic band may save fetal anatomic structures, but plastic surgeries may need to be performed after birth to correct the scar tissue in the skin. In addition to releasing the amniotic bands, the role of relaxing incisions, perpendicular to the line of scarring to minimize the constrictive effect of the scar tissues, should be explored.
We propose that the use of gas including $\mathrm{CO}_{2}$ as a distention media in the uterus allows for unprecedented visualization and access to the fetus where more complex fetal surgeries could be done such as fetal lung resection or fetal tumor excision without the need for a hysterotomy or an Exutero intrapartum treatment procedure.

\section{References}

1 Javadian P, Shamshirsaz AA, Haeri S, et al. Perinatal outcome after fetoscopic release of amniotic bands: a single-center experience and review of the literature. Ultrasound Obstet Gynecol 2013; 42(4):449-455

2 Schlehe B, Elsässer M, Bosselmann S, Axt-Fliedner R, Sohn C, Kohl T. Complete chorioamniotic membrane separation with constrictive amniotic band sequence and partial extraamniotic pregnancy: serial ultrasound documentation and successful fetoscopic intervention. J Perinatol 2014;34(12): 941-944 